\title{
Um Modelo Matemático para Mosaicagem de Imagens Digitais Baseado em uma Abordagem Ponto-a-Reta
}

\author{
A Point-to-Line Based Model for Digital Image Mosaicking
}

\author{
Francisco Pedro Soares de Lima ${ }^{1}$ \\ Daniel Rodrigues dos Santos ${ }^{2}$
}

Recebido em maio de 2019. Aprovado em novembro de 2019.

\begin{abstract}
RESUMO
Neste trabalho é proposto um modelo matemático para mosaicagem de imagens digitais. A principal característica do modelo proposto é sua abordagem ponto-a-reta, que consiste em alinhar localmente pares de imagens digitais. Os principais pontos a serem discutidos neste trabalho são: 1) extração de segmentos de retas; 2) Estimativa dos parâmetros de transformação entre pares de imagens digitais; 3) Mosaicagem digital. Primeiramente, são definidas janelas de recorte para a imagem de referência e para a imagem de pesquisa. Em seguida, segmentos de retas são extraídas empregando uma ferramenta de detecção e extração de retas, implementada por Artero (1999). Para cada janela de recorte é obtido um agrupamento ordenado de segmentos de retas. As correspondências entre o agrupamento de segmentos de retas são manualmente estabelecidas. Uma transformação matemática que mapeia cada ponto médio da linha reta extraída na imagem de referência numa linha reta correspondente na imagem de pesquisa é proposto, para estimativa dos parâmetros de transformação. Finalmente, os valores dos parâmetros de transformação estimados são introduzidos em uma matriz de construção. O resultado obtido é um mosaico de imagens digitais derivadas de um RPA (Remotely Piloted Aircraft) com transformação geométrica coincidente entre feições registradas em diferentes imagens.
\end{abstract}

PALAVRAS-CHAVE: Mosaicagem de imagens. Modelo Ponto-a-Reta. Segmentos de retas. Transformação Matemática.

\begin{abstract}
In this work a mathematical model for mosaicking of digital images is proposed. The main characteristic of the proposed model is its approach to point-to-line, which consists in aligning locally pairs of digital images. The
\end{abstract}

\footnotetext{
${ }^{1}$ Programa de Pós-graduação em Ciências Geodésicas - Universidade Federal do Paraná, Brasil. Email: lima.franciscopedrosoaresde@gmail.com

${ }^{2}$ Programa de Pós-graduação em Ciências Geodésicas - Universidade Federal do Paraná, Brasil. Email: danielsantos@ufpr.br
} 
main points to be discussed in this study are: 1) line extraction; 2) estimation of the transformation parameters between pairs of digital images; 3) Digital mosaicking. Firstly, a buffer for reference and search images are defined. Then, lines are extracted using a tool for line detection and extraction implemented by Artero (1999). For each buffer is obtained an orderly grouping of lines. The correspondences between clustered lines are manually established. A mathematical transformation that maps each mid-point of the extracted line in the reference image into a corresponding line in the search image is proposed to estimate the transformation parameters. Finally, the values of the estimated transformation parameters are introduced in a construction matrix. The result is a mosaicked digital image derived from remotely piloted aircraft (RPA).

KEYWORDS: Mosaicking. Point-to-Line Model. RPA. Mathematic transformation.

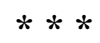

\section{Introdução}

De acordo com Xia et al. (2017), câmeras digitais têm sido amplamente empregadas para coleta de imagens aéreas, terrestres e de satélite. A partir de imagens digitais pode-se, por exemplo, construir mosaicos digitais e modelos de realidade virtual, monitorar desastres naturais, realizar estudos meteorológicos e de viabilidade de linhas de transmissão, gerar ortofotos digitais, produzir cartas topográficas, entre outros.

O emprego de mosaicos digitais por diversas vezes, se mostrou imprescindível para tomada de decisões estratégicas, principalmente, para estudos de grandes extensões territoriais. Basicamente, um mosaico digital é um produto fotogramétrico derivado da concatenação de imagens digitais tomadas com sobreposição longitudinal e lateral. A técnica empregada para geração de mosaicos digitais é conhecida como mosaicagem de imagens e pode ser dividida em duas etapas (XIA et al., 2017): 1) Alinhamento local dos pares de imagens digitais; e 2) Alinhamento global do conjunto de pares de imagens digitais. A primeira etapa consiste em estimar os parâmetros de transformação (uma rotação $\theta$, duas translações $\Delta x, \Delta y$ e dois fatores de escala $\left.\mu_{x}, \mu_{y}\right)$ entre cada par de imagens usando uma função matemática 2D $f(x, y)$ que mapeia cada ponto da imagem de referência $I(x, y)$ a um ponto 
correspondente na imagem de pesquisa $I^{\prime}(x, y)$. A segunda etapa consiste em refinar a estimativa dos parâmetros de transformação e materializar um sistema referencial único para o mosaico digital. Neste trabalho, será dado ênfase apenas para a primeira etapa, isto é, a técnica de alinhamento local dos pares de imagens digitais.

O alinhamento local de pares de imagens digitais é tarefa crucial para a construção de mosaicos digitais, além de influenciar diretamente a qualidade do produto gerado. Geralmente, as abordagens empregadas para estimativa dos parâmetros de transformação de pares de imagens digitais são agrupadas em modelos de correspondência por área e modelos de correspondência por feições. De acordo com Schenk (1999) a correspondência por área compara a distribuição de níveis de cinza de uma subimagem $S(x, y)$ com sua correspondente em $I^{\prime}(x, y)$ e pode ser feito usando funções matemáticas que determinam seu coeficiente de correlação ou através da minimização da diferença de níveis de cinza entre $S(x, y)$ e uma janela de correlação em $I^{\prime}(x, y)$. Para Ali et al. (2016), o alto custo computacional da técnica de correspondência por área inviabiliza seu emprego em tarefas de mosaicagem de imagens. Já as abordagens de correspondência baseadas em feições, também conhecida como correspondência por característica ou correspondência estrutural, são técnicas que realizam o processamento localmente, ou seja, em regiões de pixels de borda e de canto. $\mathrm{O}$ objetivo deste processo é encontrar o melhor mapeamento entre descritores locais, sendo necessárias três subtarefas, a saber: A) Detecção e extração de feições (pontos ou segmentos de retas); B) Construção dos descritores relacionais; e C) Estabelecimento das correspondências. O SIFT (Scale Invariant Feature Transform) é o algoritmo mais empregado para as tarefas de detecção e extração de feições, construção de descritores relacionais e estabelecimento de suas correspondências (LOWE, 2004), que se têm notícias e foi proposto em 2004 por David Lowe.

Usualmente, o modelo matemático adotado para mapear as primitivas entre as imagens depende da característica do método de correspondência 
empregado. Por exemplo, se feições pontuais são detectadas e extraídas para encontrar o melhor mapeamento entre seus descritores locais o método de correspondência usado é baseado em uma abordagem ponto-a-ponto. Enquanto as feições pontuais são largamente empregadas em tarefas de construção de mosaicos digitais por serem abundantes e não se degeneram ao serem projetadas (GALO e TOZZI, 2004), os segmentos de retas estão presentes em ambientes modificados pelo homem, são perenes, não requer correspondência ponto-a-ponto, são fáceis de serem extraídas em imagens digitais e são menos suscetíveis a falsos positivos no estabelecimento de suas correspondências (LIU et al., 1990; DAL POZ et al., 1996; ROBERTS, 1988; TOMMASELLI e LUGNANI, 1988; TOMMASELLI e TOZZI, 1996; KUBIK, 1988; BUCHANAN, 1992; PETSA e PATIAS, 1994; HEUVEL, 1997; HABIB, 1999; ZALMASON, 2000; HABIB e SCHENK, 1999; HABIB et al., 2005; SHAN, 2001; KLINEC, 2004; ZHANG, 2004).

Neste trabalho, será investigado um modelo matemático para mosaicagem de imagens digitais baseado em uma abordagem ponto-a-reta. A principal contribuição do método proposto é o desenvolvimento de uma transformação matemática que mapeia cada ponto médio da linha reta extraída na imagem de referência $I(x, y)$ a uma linha reta correspondente na imagem de pesquisa $I^{\prime}(x, y)$, para estimativa dos parâmetros de transformação (alinhamento local).

\section{Estado da Arte em Mosaicagem de Imagens Digitais}

Basicamente, os métodos existentes de geração de mosaicos digitais são proeminentemente baseados em abordagens ponto-a-ponto. Neste trabalho os métodos serão agrupados em duas principais categorias, como segue:

a) Baseado em caraterísticas (ou feições pontuais): Xing et al. (2010) apresentaram um modelo de otimização em duas etapas para mosaicagem de imagens sequenciais adquiridas por uma plataforma RPA. Os autores usaram o algoritmo SURF para detecção e extração de feições pontuais, construir os 
descritores relacionais, estabelecer as correspondências e o filtro de Kalman para estimar os parâmetros de transformação. Li et al. (2012) propuseram uma estratégia para registro e mosaicagem de dados RPA. Os autores empregaram o algoritmo SIFT para o processo de correspondência e o algoritmo RANSAC para detectar e remover falsas correspondências de pontos. Os parâmetros de transformação foram estimados usando um ajustamento simultâneo de feixes de raios de luz perspectivos. A otimização dos valores de translação e rotação foi realizada com a aplicação do método de Levenberg-Marquardt. Gao e Jia (2007), desenvolveram método automático para mosaicagem de pares de imagens. Para determinação das primitivas utilizou-se o algoritmo de Harris e aplicou-se RANSAC para o refinamento das correspondências. Os parâmetros de transformação foram estimados utilizando método de Levenberg-Marquardt. Nemra e Aouf (2009) propuseram um método de mosaicagem de imagens de alta resolução. Para descrição e detecção de primitivas utilizaram o algoritmo SIFT. Aplicaram uma transformação geométrica (transformação de similaridade associada a uma transformação afim) para estimar os valores de rotação e translação em uma abordagem ponto-a-ponto. Cho et al. (2003) também propuseram uma abordagem ponto-a-ponto para a resolução do mesmo problema. Os autores aplicaram o MMQ para estimar os parâmetros de transformação entre pares de imagens, utilizando dois modelos matemáticos: transformação afim e biquadrática. Para construção de mosaico digital, Islam e Kabir (2013) propuseram uma técnica de registro de imagens. Para esta tarefa, usaram operador de Sobel com um par de Kernel de convolução 3x3 para eliminar ruídos. Posteriormente, realizaram a segmentação de bordas de objetos presentes na imagem e as utilizaram como primitivas. A translação entre bordas homólogas extraídas de diferentes imagens é estimada a partir do cálculo da distância de Hausdorff. Para eliminar as correspondências incorretas basearam-se na preservação da distância entre dois pontos da mesma imagem, após aplicação de uma transformação rígida. Xiao et al. (2005) propuseram um método de expansão de região para propagar de forma adaptável o alinhamento de regiões, aplicaram algoritmo de base longa para calcular um conjunto de correspondência e um registo robusto foi alcançado com base em caraterísticas de 
alto nível. Tian et al. (2003) propuseram um método para mosaicagem automática de imagens digitais baseado em pontos de interesse. Tian et al. (2018) propuseram um método de mosaicagem de imagens baseado na determinação de linha de costura, buscando área mínima de conexão e árvore de expansão mínima para eliminar a descontinuidade visual observável e melhorar a eficiência do tempo. Elibol et al. (2013) propuseram uma abordagem em grafos para mosaicagem de imagens, neste metodo as imagens são definidas como nós e as correspondências entre pares de imagens são definidas como arestas. Com utilização desta estrutura, o método é capaz de obter correspondências entre as imagens com um número reduzido de tentativas, diminuindo custo computacional. Xia et al. (2017) propuseram uma abordagem em grafos para o alinhamento globalmente consistente de imagens obtidas de cenas aproximadamente planares, através de análise topológica.

b) Baseado em área: Berberidis et al. (2002) propuseram um algoritmo para registro de pares de imagens. A técnica consiste na aplicação do metodo de correlação cruzada para estimativa dos valores de translação e rotação. Em um processo iterativo o algoritmo refina os parâmetros de transformação até que os pares de imagens sejam concatenados em um mesmo referencial. Ghannam e Abbott (2013) propuseram um método baseado em correlação cruzada combinada com a técnica da média de pixels, em que cada pixel no mosaico visa extrair seu valor em apenas uma única imagem. Patidar e Jain (2011) utilizaram um esquema baseado em correlação de imagens que opera no dominio de Fourier para encontrar os parâmetros de transformação entre pares de imagens e empregá-los no processo de mosaicagem. 0 Quadro 1 mostra as vantagens e desvantagens de cada um dos métodos supracitados. 
Quadro 1 - Vantagens e desvantagens dos métodos de alinhamento local de imagens digitais

\begin{tabular}{|c|c|c|c|}
\hline Categorias & Vantagem & Desvantagem & Abordagem \\
\hline (a) & $\begin{array}{l}\text { - Correspondência } \\
\text { precisa. } \\
\text { - Fácil alinhamento. } \\
\text { - Várias aplicações. } \\
\text { - Computação simples, } \\
\text { precisa e rápida. }\end{array}$ & $\begin{array}{l}\text { - Grande consumo de } \\
\text { memória. } \\
\text { - Inconsistência } \\
\text { geométrica. } \\
\text { - Computacionalmente } \\
\text { caro. } \\
\text { - A precisão depende de } \\
\text { grandes sobreposições. }\end{array}$ & $\begin{array}{l}\text { Ponto-a- } \\
\text { ponto }\end{array}$ \\
\hline (b) & $\begin{array}{l}\text { - Regiões das imagens } \\
\text { são usadas para estimar } \\
\text { a correspondência. } \\
\text { - Menos sensível a } \\
\text { mudanças de } \\
\text { iluminação e oclusão. }\end{array}$ & $\begin{array}{l}\text { - Resultado incorreto. } \\
\text { - Raramente usados. } \\
\text { - Maior tempo } \\
\text { computacional. } \\
\text { - Inconsistência } \\
\text { geométrica. }\end{array}$ & $\begin{array}{l}\text { Ponto-a- } \\
\text { ponto }\end{array}$ \\
\hline
\end{tabular}

Fonte: Elaborado pelos autores.

\section{Método Proposto para Mosaicagem de Imagens Digitais}

Este trabalho trata do alinhamento local de pares de imagens para mosaicagem de imagens digitais usando um modelo matemático baseado em uma abordagem ponto-a-reta. 
Figura 1 - Arquitetura do método proposto para mosaicagem de imagens digitais

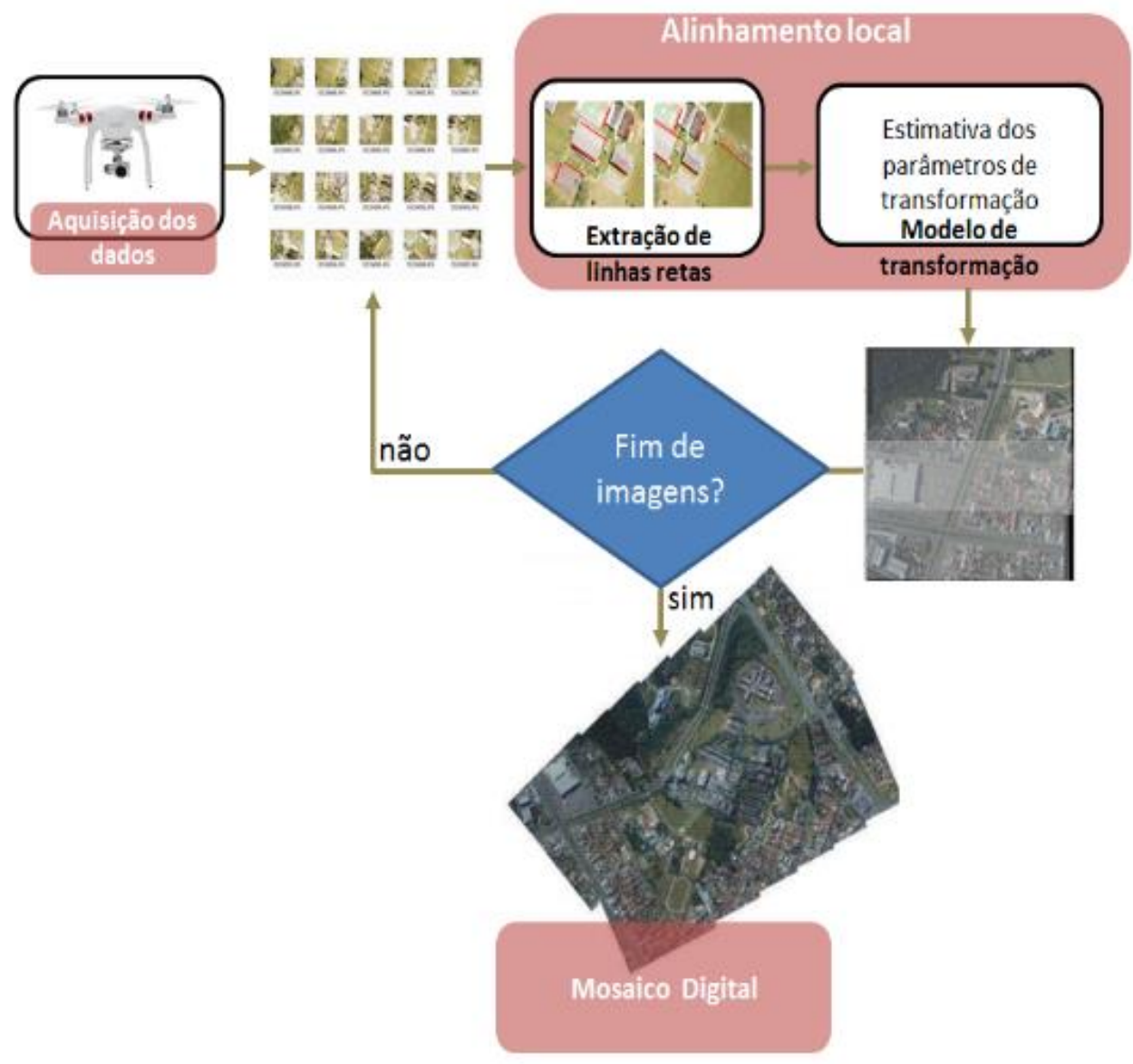

Fonte: Elaborada pelos autores.

0 alinhamento local consiste em alinhar pares de imagens digitais, consistindo de três etapas: 1) Extração de segmentos de retas em pares de imagens digitais e estabelecimento de suas correspondências; 2) Estimativa dos parâmetros de transformação entre pares de imagens; e 3) Geração do mosaico digital. A Figura 1 mostra a arquitetura das etapas envolvidas no desenvolvimento deste trabalho. De acordo com a Figura 1, primeiramente é necessário obter as imagens aéreas usando um sensor passivo embarcado em uma plataforma móvel aérea, tal como um RPA (Remotely Piloted Aircraft). Em seguida, para cada par de imagens digitais é aplicado o algoritmo de extração de feições retas e estabelecido suas correspondências. Posteriormente, os parâmetros de transformação (alinhamento local) são estimados usando o modelo de transformação proposto. Finalmente, o mosaico digital é gerado. A seguir serão detalhadas as etapas do método proposto. 
3.1 Pré-processamento dos dados de entrada

Dado um par de imagens $I(x, y)$ e $I^{\prime}(x, y)$, como apresentado na Figura 2, a primeira etapa do método proposto neste trabalho consiste em definir janelas de recorte, $J_{i}$ para a imagem de referência $I(x, y)$ e $J_{j}$ para a imagem de pesquisa $I^{\prime}(x, y)$. Em seguida, segmentos de retas são extraídas em $J_{i}$ e $J_{j}$ empregando uma ferramenta de detecção e extração de segmentos de retas. O algoritmo implementado segue a seguinte sequência de etapas: A) Suavização da imagem usando o filtro mediana; B) Segmentação da imagem através do operador Sobel e limiarização por OTSU; e C) Afinamento de bordas por supressão não-máxima. O algoritmo de extração de segmentos de retas é derivado do trabalho de Artero (1999). A Figura 2 mostra a arquitetura da etapa de pré-processamento dos pares de imagens.

Figura 2 - Etapas do pré-processamento dos dados

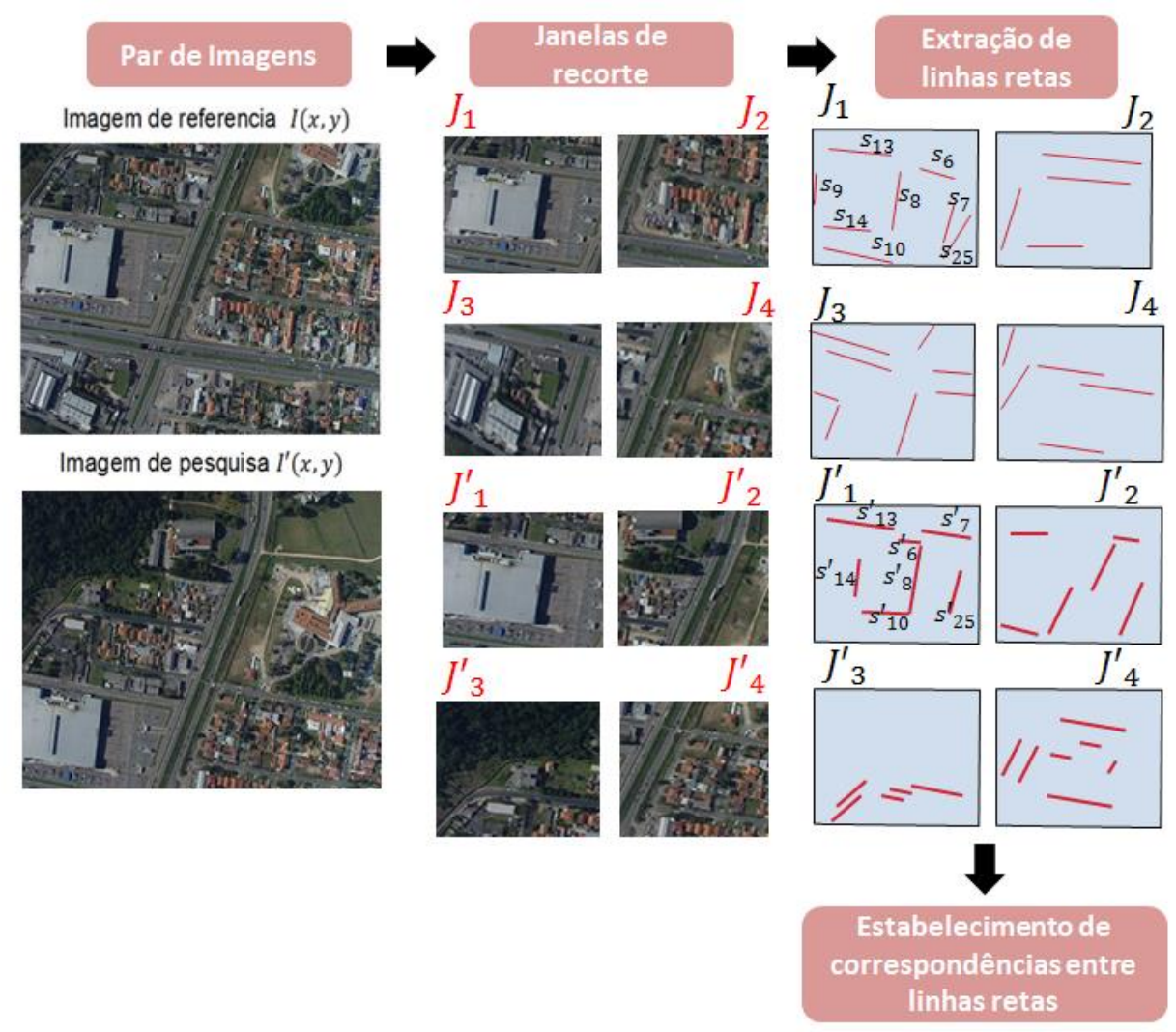

Fonte: Elaborada pelos autores. 
Para cada janela de recorte $J_{i}$ e $J_{j}$ é obtido um agrupamento ordenado de segmentos de retas $\left(l_{i}, l_{j}^{\prime}\right)$, sendo $l_{i}$ o agrupamento de segmentos de retas extraídas em $J_{i}$ e $l_{j}^{\prime}$ o agrupamento de segmentos de retas extraídas em $J_{j}$, ambos no sistema referencial digital:

$$
\begin{aligned}
l_{i} & =\left\{s_{1}, s_{2}, \ldots, s_{M}\right\} \\
l^{\prime}{ }_{j} & =\left\{s^{\prime}{ }_{1}, s^{\prime}{ }_{2}, \ldots, s^{\prime}{ }_{N}\right\}
\end{aligned}
$$

sendo, $s_{M}$ e $s_{N}^{\prime}$ a i-ésima linha reta em $J_{i}$ e $J_{j}$, respectivamente para $M>N$.

Na Figura 2, considere que as correspondências entre o agrupamento de segmentos de retas $l_{1}=\left\{s_{6}, s_{7}, s_{8}, s_{9}, s_{10}, s_{13}, s_{14}, s_{25}\right\}$ presente na janela de recorte $J_{1}$ e o agrupamento $l_{1}^{\prime}=\left\{s_{6}{ }_{6}, s^{\prime}{ }_{7}, s^{\prime}{ }_{8}, s_{9}{ }_{9}, s^{\prime}{ }_{10}, s^{\prime}{ }_{13}, s_{14}^{\prime}, s^{\prime}{ }_{25}\right\}$ contido na janela de recorte $J^{\prime}{ }_{1}$ foram manualmente estabelecidas. Vale ressaltar que somente os valores do ponto entre as extremidades dos segmentos de retas de $l_{1}\left(p_{i}=[x, y]^{T}\right)$ e os parâmetros $a, b, c$ de cada linha reta de $l_{1}^{\prime}$ correspondente são armazenados. Todo o procedimento descrito anteriormente é aplicado para todos os agrupamentos extraídos nas janelas de pesquisas $\left(J_{2}, J_{3}, J_{4}, J_{2}^{\prime}, J^{\prime}{ }_{3}, J^{\prime}{ }_{4}\right)$. O resultado é um conjunto de pares de segmentos de retas correspondentes.

Para alinhar localmente um par de imagens digitais é necessário estimar os parâmetros de transformação que descreve uma rotação, duas translações e um fator de escala uniforme entre as imagens.

\subsection{Alinhamento local de pares de imagens digitais}

Neste trabalho é proposta uma transformação matemática que mapeia cada ponto $p=[x, y]^{T}$ entre as extremidades da linha reta extraída na imagem de referência numa linha reta $l=[a, b, c]^{T}$ correspondente na imagem de pesquisa para estimativa dos parâmetros de transformação. O simples deslocamento da origem da imagem I para uma nova imagem I' é considerada uma transformação matemática. Essa transformação pode ser escrita como uma função vetorial dada na forma (HARTLEY e ZISSERMAN, 2000): 


$$
I^{\prime}=f(I), f: \mathbb{I R}^{2} \mapsto \mathbb{R}^{2}
$$

Uma transformação afim geral no plano é uma transformação geométrica que preserva colinearidade entre os pontos e a razão de distância entre eles. Basicamente, uma transformação afim descreve dois fatores de escala $\left(\mu_{x}, \mu_{y}\right)$, uma rotação $(\theta)$, duas translações $\left(\mathrm{t}_{\mathrm{x}}, \mathrm{t}_{\mathrm{y}}\right)$ e um fator de não ortogonalidade $(\delta)$.

De forma geral, uma matriz de transformação $H$ pode ser construída por uma sequência de transformações afim, desde que as operações matriciais sejam realizadas por meio de coordenadas homogêneas. Por exemplo, considere três matrizes de transformação $T_{1}$ (translação), $T_{2}$ (fator de escala) e $T_{3}$ (rotação):

$$
T_{1}=\left[\begin{array}{lll}
1 & 0 & t_{x} \\
0 & 1 & t_{y} \\
0 & 0 & 1
\end{array}\right], T_{2}=\left[\begin{array}{ccc}
\mu_{x} & 0 & 0 \\
0 & \mu_{y} & 0 \\
0 & 0 & 1
\end{array}\right], T_{3}=\left[\begin{array}{ccc}
\cos \theta & \operatorname{sen} \theta & 0 \\
-\operatorname{sen} \theta & \cos \theta & 0 \\
0 & 0 & 1
\end{array}\right]
$$

Neste caso, a matriz $H$ pode ser obtida multiplicando $T_{1}, T_{2}$ e $T_{3}$ na seguinte ordem, a saber:

$$
H=T_{3} T_{2} T_{1}=\left[\begin{array}{ccc}
\mu_{x} \cos \theta & \mu_{y} \operatorname{sen} \theta & {\left[\mu_{x}\left(t_{x} \cos \theta\right)+\mu_{y}\left(t_{y} \operatorname{sen} \theta\right)\right]} \\
-\mu_{x} \operatorname{sen} \theta & \mu_{y} \cos \theta & {\left[\mu_{y}\left(t_{y} \cos \theta\right)-\mu_{x}\left(t_{x} \operatorname{sen} \theta\right)\right]} \\
0 & 0 & 1
\end{array}\right]
$$

Seja um ponto $p=[x, y, 1]^{T}$, em coordenadas homogêneas, pertencente à linha reta $l=[a, b, c]^{T}$, uma vez que $a x+b y+c=0$. Esta relação pode ser escrita em termos do produto interno entre vetores, como segue:

$$
(x, y, 1)^{T}\left[\begin{array}{l}
a \\
b \\
c
\end{array}\right]=p^{T} l=0
$$

sendo $a, b$ e $c$ os parâmetros de $l$.

Introduzindo a matriz $H$ na Equação (6) resulta o modelo de transformação matemática proposto neste trabalho, como segue:

$$
\begin{gathered}
p^{T} H l=0 \\
(x, y, 1)^{T}\left[\begin{array}{ccc}
\mu_{x} \cos \theta & \mu_{y} \operatorname{sen} \theta & {\left[\mu_{x}\left(t_{x} \cos \theta\right)+\mu_{y}\left(t_{y} \operatorname{sen} \theta\right)\right]} \\
-\mu_{x} \operatorname{sen} \theta & \mu_{y} \cos \theta & {\left[\mu_{y}\left(t_{y} \cos \theta\right)-\mu_{x}\left(t_{x} \operatorname{sen} \theta\right)\right]} \\
0 & 0 & 1
\end{array}\right]\left[\begin{array}{l}
a \\
b \\
c
\end{array}\right]=0
\end{gathered}
$$

A partir da Equação (8) a matriz $H$ é determinada em função de um ponto $p=[x, y, 1]^{T}$ entre as extremidades da linha reta extraída em $I(x, y)$ e uma linha reta $l=[a, b, c]^{T}$ correspondente em $I^{\prime}(x, y)$. Como o modelo 
matemático proposto é não linear e as observações e os parâmetros atendem uma injunção de condição, a solução do problema é feito através do modelo combinado do MMQ (Método dos Mínimos Quadrados). De acordo com Mikhail e Ackerman (1976) o método combinado é aplicado em modelos funcionais que ajustam observações e parâmetros. Os modelos funcionais aplicados a este método são formados por equações implícitas do tipo:

$$
F\left(X_{a}, L_{a}\right)=0
$$

$\mathrm{O}$ método combinado de ajustamento não-linear é obtido através da linearização utilizando a expansão em série de Taylor, tomando-se apenas os dois primeiros termos da série, assim:

$$
A X+B V+W=0
$$

sendo $A=\left.\frac{\partial F}{\partial X_{a}}\right|_{X_{0}, L_{b}}, B=\left.\frac{\partial F}{\partial L_{a}}\right|_{X_{0}, L_{b}}, W=F\left(X_{0}, L_{b}\right), X=X_{a}-X_{0}, V=L_{a}-L_{b}, X_{0}$ é o vetor dos parâmetros aproximados, $X_{a}$ é o vetor dos parâmetros ajustados $L_{b}$ é o vetor onde são inseridos as observação e $L_{a}$ é o vetor das observações ajustadas. Desta forma, tem-se a seguinte solução para as equações normais.

$$
X=\left[A^{T}\left(B P B^{T}\right)^{-1} A\right]^{-1} A^{T}\left(B P B^{T}\right)^{-1} W
$$

Desde que os parâmetros de transformação são estimados pelo MMQ o processo de mosaicagem para cada par de imagens digitais é realizado.

\subsection{Mosaicagem de imagens digitais}

Para a mosaicagem de imagens digitais, os valores estimados de escala $\left(\mu_{x}, \mu_{y}\right)$, rotação $(\theta)$ e translação $\left(\mathrm{t}_{\mathrm{x}}, \mathrm{t}_{\mathrm{y}}\right)$ são introduzidos em uma matriz de construção $(Q)$, como segue:

$$
Q=\left[\begin{array}{ccc}
r_{x} & q_{x} & f_{x} \\
-q_{y} & r_{y} & f_{y} \\
0 & 0 & 1
\end{array}\right]
$$

sendo $r_{x}=\mu_{x} \cos \theta, r_{y}=\mu_{y} \cos \theta, q_{x}=\mu_{x} \operatorname{sen} \theta, q_{y}=\mu_{y} \operatorname{sen} \theta, f_{x}=\mu_{x}\left(t_{x} \cos \theta\right)+$ $\mu_{y}\left(t_{y} \operatorname{sen} \theta\right), f_{y}=\mu_{y}\left(t_{y} \cos \theta\right)-\mu_{x}\left(t_{x} \operatorname{sen} \theta\right)$. 
Seja $X=\left[\begin{array}{ll}x & y\end{array}\right]^{T}$ um ponto qualquer na origem de pesquisa aplicando a matriz $Q$ o ponto $\mathrm{X}$ será transformado para $\mathrm{X}^{\prime}=\left[\mathrm{x}^{\prime} \mathrm{y}^{\prime} 1\right]^{\mathrm{T}}$ na imagem de referência, como segue:

$$
X^{\prime}=Q \cdot X
$$

Aplicando a Equação (13) para todos os pontos da imagem de pesquisa o mosaico será construído. No entanto, como os valores de pixel são diferentes de cada imagem é necessário aplicar uma interpolação bilinear. O resultado é um mosaico com uma transformação geométrica coincidente de feições entre as imagens. A seguir serão apresentados os experimentos e a discussão dos resultados.

\section{Experimentos e Discussão dos Resultados}

Para avaliar o potencial do modelo matemático proposto, neste trabalho, foram realizados quatro cenários usando imagens digitais coletadas por uma câmera digital de pequeno formato modelo Sony Nex-6 embarcada em um RPA profissional multirotor (6 hélices) da marca DJI, modelo Spreading Wings S800. As imagens cobrem uma determinada área de teste do centro politécnico da UFPR. Os pares de imagens foram tomados com uma sobreposição longitudinal de $70 \%$ e lateral $60 \%$. 
Figura 3 - Pares de imagens do cenário I. (a) (c) (e) imagens de referência para os experimentos 1,2 e 3; (b) (d) (f) imagens de pesquisa para os experimentos 1,2 e 3

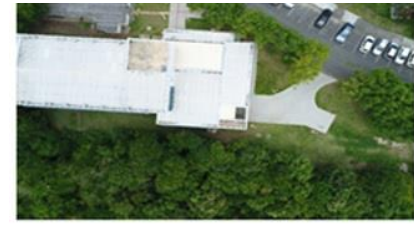

(a) Imagem de referência I $(x, y)$

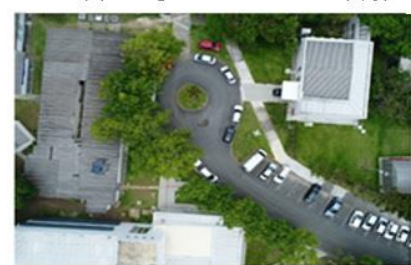

(c) Imagem de referência $I(x, y)$

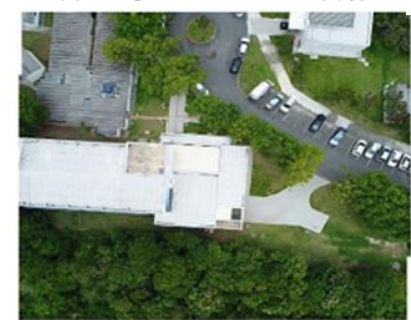

(e) Imagem de referência I(x, y)

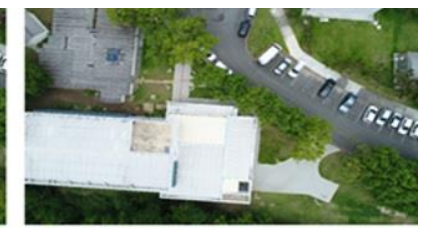

(b) Imagem de pesquisa $\mathrm{I}^{\prime}(\mathrm{x}, \mathrm{y})$

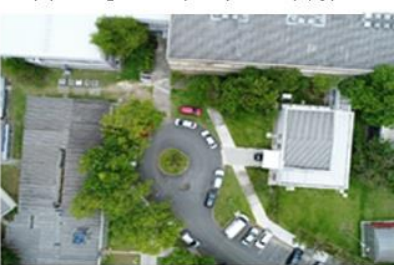

(d) Imagem de pesquisa $I^{\prime}(x, y)$

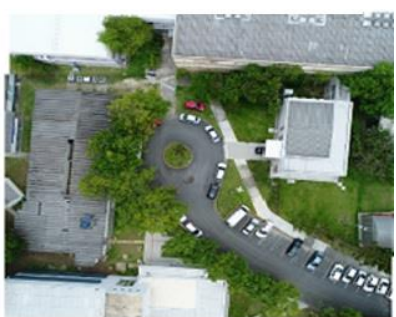

(f) Imagem de pesquisa $I^{\prime}(x, y)$

Fonte: Elaborada pelos autores.

Como mostrado na Figura 3, o primeiro cenário de experimentos contém três pares de imagens digitais, que são compostas por edificações altas e baixas, arruamento, vegetação, faixas de trânsito, automóveis, entre outras.

Como descrito anteriormente, segmentos de retas são extraídas usando o algoritmo implementado e suas correspondências são manualmente estabelecidas. Os pontos entre as extremidades $\left(x_{m}, y_{m}\right)$ dos segmentos de retas são extraídas na imagem de referência manualmente, enquanto na imagem de pesquisa os parâmetros $a, b, c$ de cada linha reta correspondente são determinados usando ajustamento de observações como apresentado em Artero (199). A Tabela 2 mostra as coordenadas dos pontos médios e os parâmetros dos segmentos de retas correspondentes coletadas nas imagens de referência e de pesquisa. 
Tabela 2 - Coordenadas dos pontos e os parâmetros dos segmentos de retas correspondentes coletadas nas imagens de referência e de pesquisa

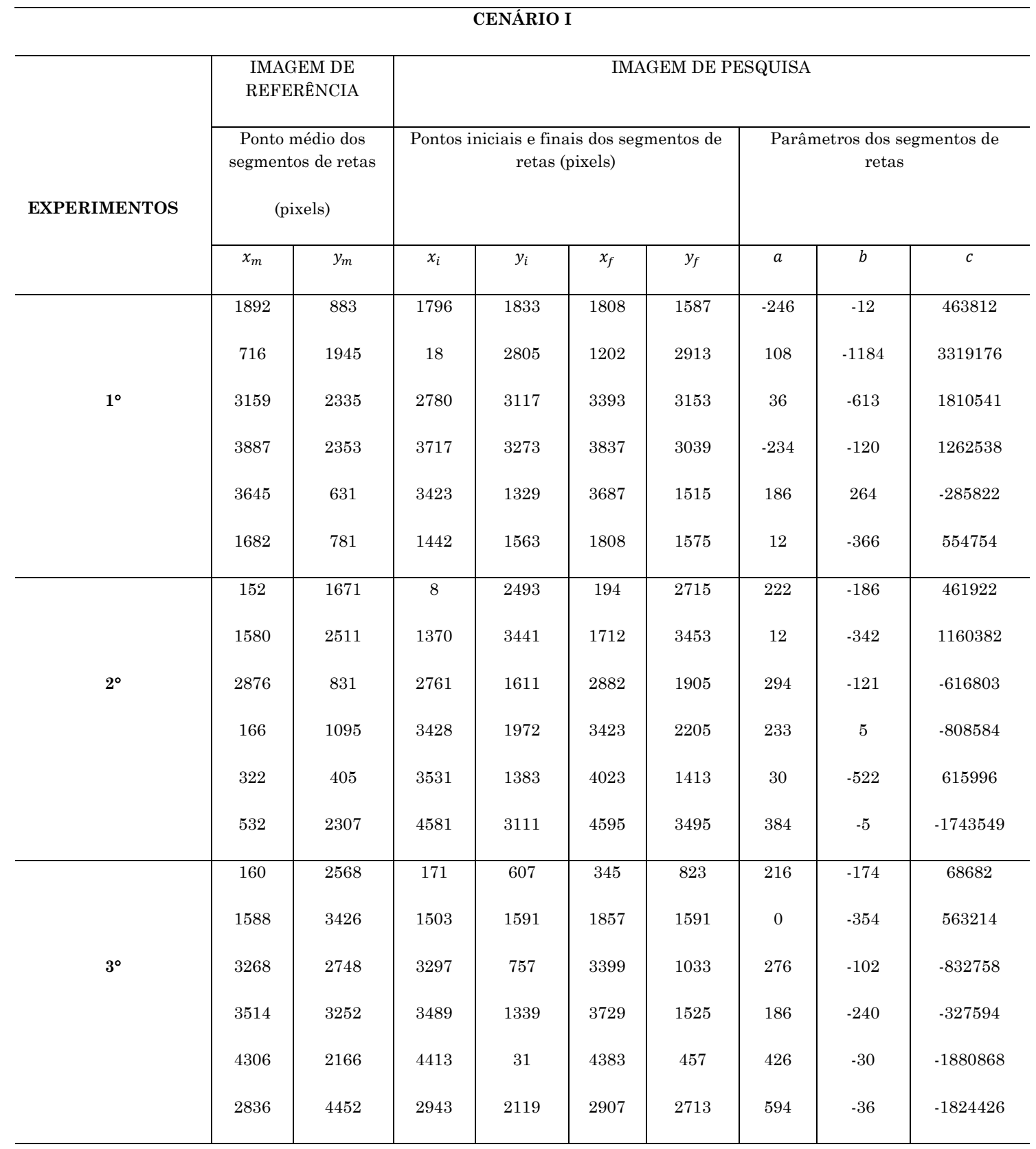

Fonte: Elaborada pelos autores.

Os dados apresentados na Tabela 2 foram usados no modelo matemático proposto neste trabalho (Eq. 8) e os parâmetros de transformação $\left(\mu_{x}, \mu_{y}, \theta, \mathrm{t}_{\mathrm{x}}, \mathrm{t}_{\mathrm{y}}\right)$ estimados pelo MMQ. A Tabela 3 mostra os parâmetros de transformação estimados com o modelo proposto. 
Tabela 3 - Parâmetros de transformação estimados pelo modelo proposto

\begin{tabular}{|c|c|c|c|c|}
\hline \multirow[t]{2}{*}{ PARÂMETROS } & \multirow[t]{2}{*}{$\begin{array}{l}\text { VALORES } \\
\text { INICIAIS }\end{array}$} & $\begin{array}{c}\text { EXPERIMENTO } \\
1\end{array}$ & EXPERIMENTO 2 & $\begin{array}{c}\text { EXPERIMENTO } \\
3\end{array}$ \\
\hline & & Valores estimados & Valores estimados & Valores estimados \\
\hline $\boldsymbol{\theta}$ (rad.) & 0.05 & -0.0487 & 0.0135 & 0.0025 \\
\hline $\boldsymbol{t}_{\boldsymbol{x}}$ (pixel) & 0 & 45.3923 & 60.4233 & -86.6924 \\
\hline $\boldsymbol{t}_{\boldsymbol{y}}$ (pixel) & 0 & -939.3526 & -933.0041 & 1854 \\
\hline$\mu_{x}$ & 1 & 1 & 1 & 1 \\
\hline$\mu_{y}$ & 1 & 1 & 1 & 1 \\
\hline
\end{tabular}

Fonte: Elaborada pelos autores.

Como pode ser observado na Tabela 3, os parâmetros de translação $\left(t_{x}, t_{y}\right)$ estimados pelo método proposto através dos experimento 1,2 e 3 (a diante designado por E1, E2 e E3) apresentam os movimentos gerais de cada experimento que deve ser $E 1(45.3923,-939.3526), E 2(60.4233,-933.0041)$ e $E 3(-86.6924,1854)$ respetivamente; quanto aos fatores de escala $\left(\mu_{x}, \mu_{y}\right)$ estimados, são iguais a 1 para todos os experimentos, indicam que não houve mudanças de escalas das imagens. Em relação aos valores de rotação $(\theta)$ estimados $E 1(\theta=-0.0487 \mathrm{rad}), E 2(\theta=0.0135 \mathrm{rad})$ e $E 3(\theta=0.0025 \mathrm{rad})$, o valor inicial para todos os experimentos foi $\theta=0.05 \mathrm{rad}$. Aplicando os valores estimados com o modelo apresentado na Eq. (13) é obtido o mosaico digital para cada par de imagens digitais do Cenário I, como mostrado a Figura 4.

$\mathrm{Na}$ Figura 4 são apresentadosi os mosaicos digitais obtidos com o método proposto usando os pares de imagens dos experimentos 1 e 2 . Note que não há diferença radiométrica significativa em ambos os mosaicos digitais. Além disso, as inconsistências geométricas entre os segmentos de de junção das feições cartográficas não são perceptíveis. 
Figura 4 - Mosaicos digitais obtidos com o método proposto. (a) Mosaico digital do experimento 1. (b) Mosaico digital do experimento 2

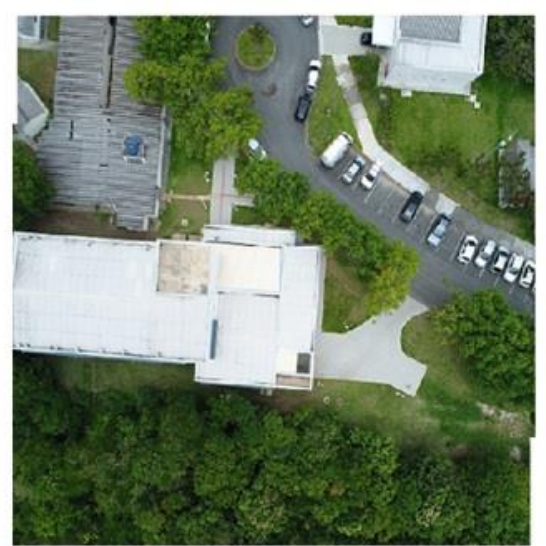

(a)

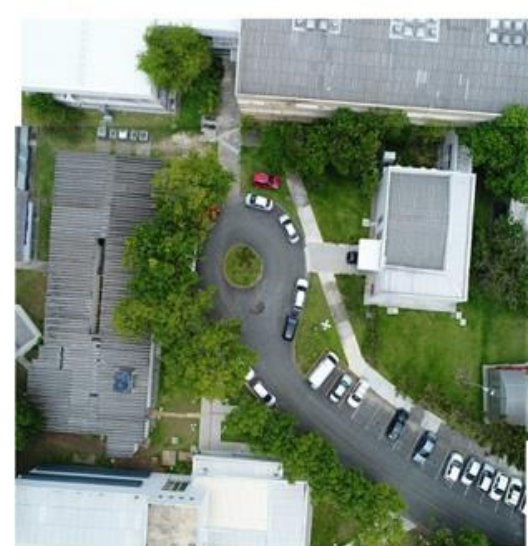

(b)

Fonte: Elaborada pelos autores.

Na Figura 5 é apresentado o mosaico digital produzido com as imagens dos pares referentes aos experimentos 1 e 2. É possível perceber uma linha, no canto inferior direito, que separa geometricamente as feições cartográficas. Este efeito foi produzido pela falta da presença de segmentos de retas nesta região da imagem dificultando a estimativa dos parâmetros de transformação. No pré-processamento foram coletados seis segmentos de retas nas posições das bordas de objetos presentes em cada imagem de entrada. 
Figura 5 - Mosaicos digitais obtidos com o método proposto. (a) Mosaico digital do experimento 3. (b) Recorte do mosaico e ampliação das regiões

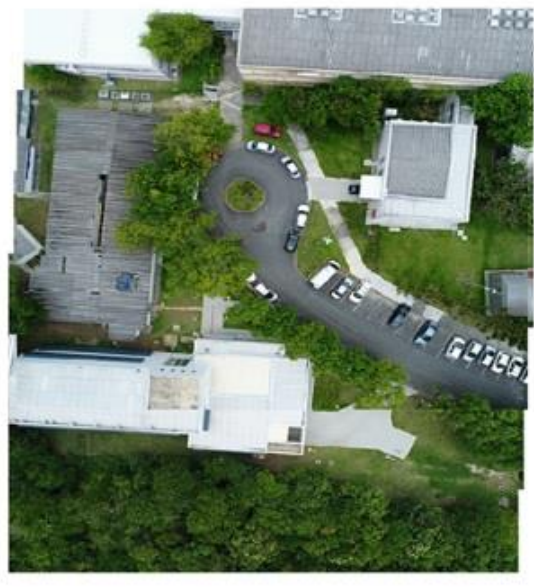

(a)

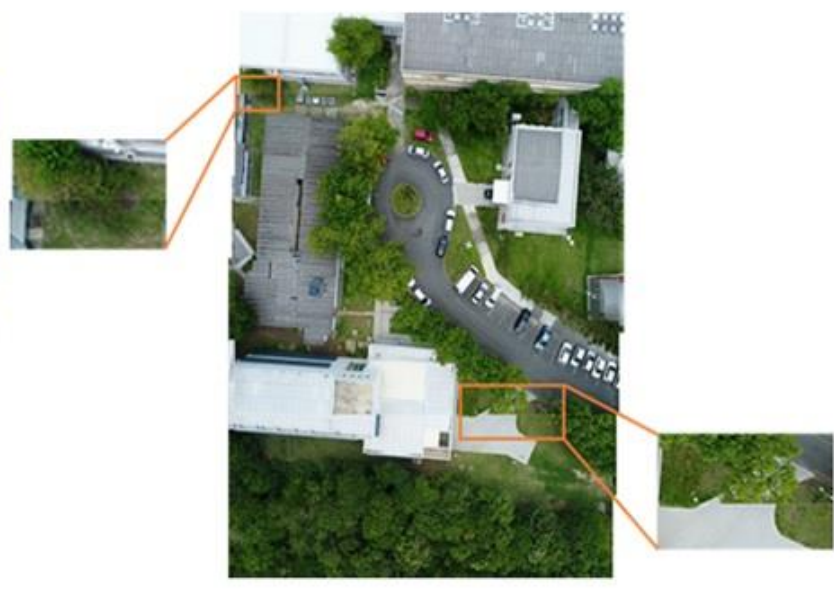

(b)

Fonte: Elaborada pelos autores.

As descontinuidades encontradas no mosaico apresentado na Figura $5 b$, também podem ser causadas pelas regiões com grandes alterações radiométricas devido à mudança de ponto de vista do sensor e variação de iluminação e pelos erros na extração dos segmentos de retas. Uma forma de melhorar os resultados obtidos é empregando técnicas de feathering, como descrito em Oliveira et al. (2018). O mesmo procedimento descrito anteriormente foi adotado para os Cenários II e III. A Figura 6 mostra as imagens de referência e de pesquisa usadas nos experimentos do Cenário II. 
Figura 6 - Pares de imagens do cenário II. Imagens de referência e de pesquisa para (a) Experimento 4. (b) Experimento 5. (c) Experimento 6

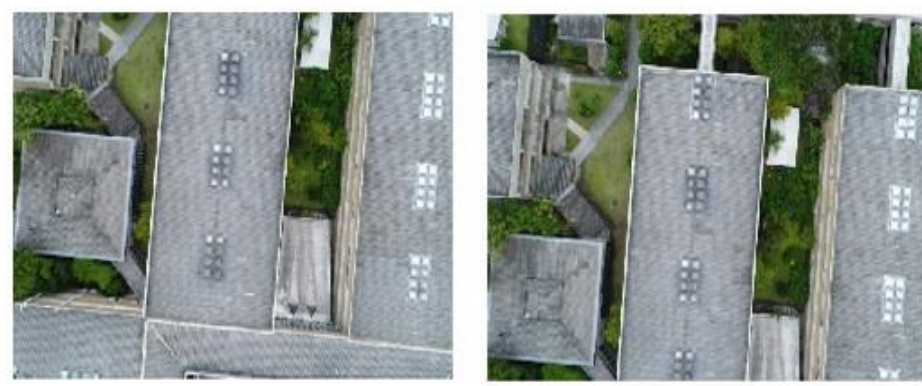

(a)
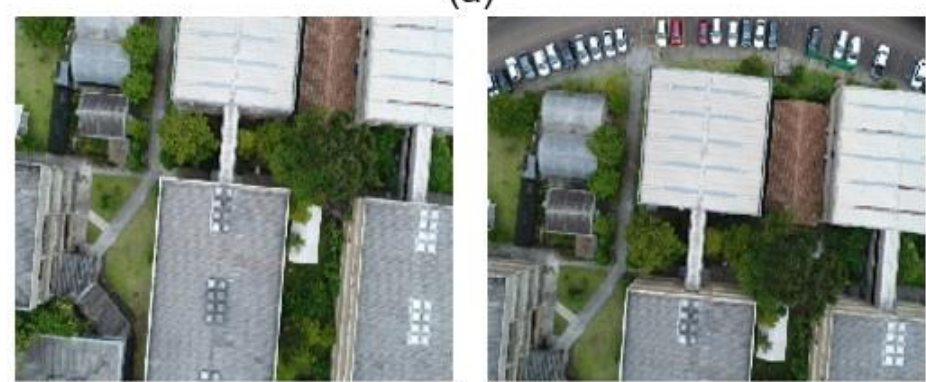

(b)
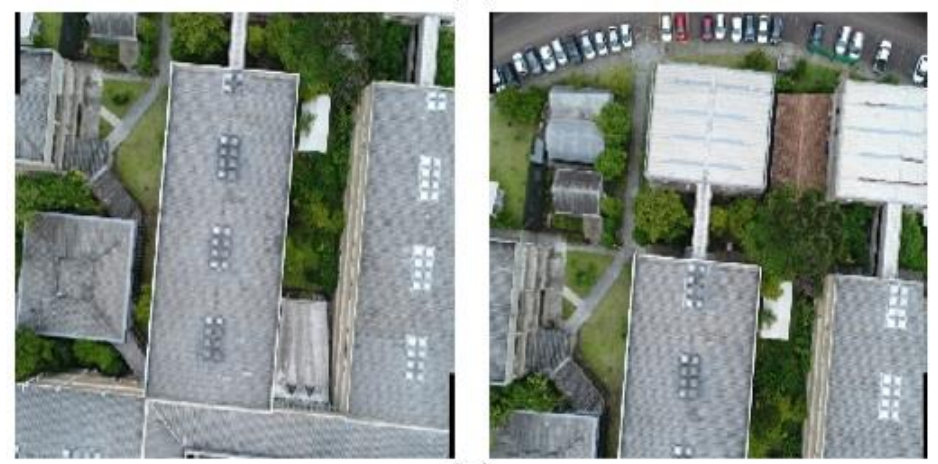

(c)

Fonte: Elaborada pelos autores.

A Figura 7 mostra os mosaicos digitais obtidos com o método proposto para cada experimento do Cenário II. 
Figura 7 - Mosaicos digitais obtidos com o método proposto. (a) Mosaico digital do experimento 4. (b) Mosaico digital do experimento 5. (c) Mosaico digital do experimento 6

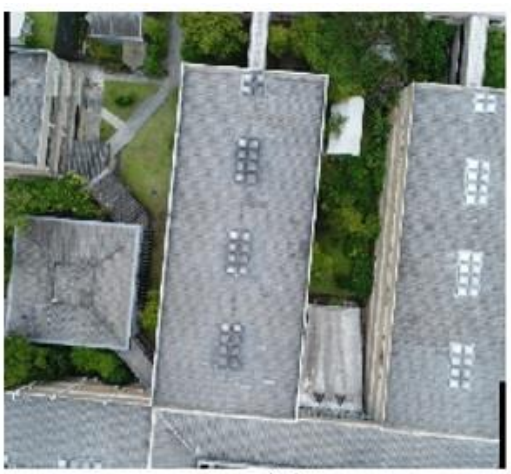

(a)

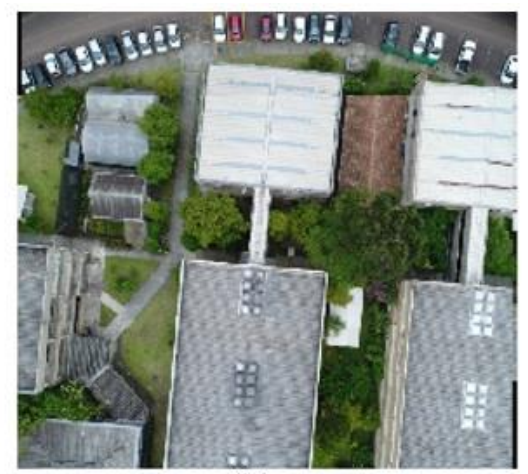

(b)

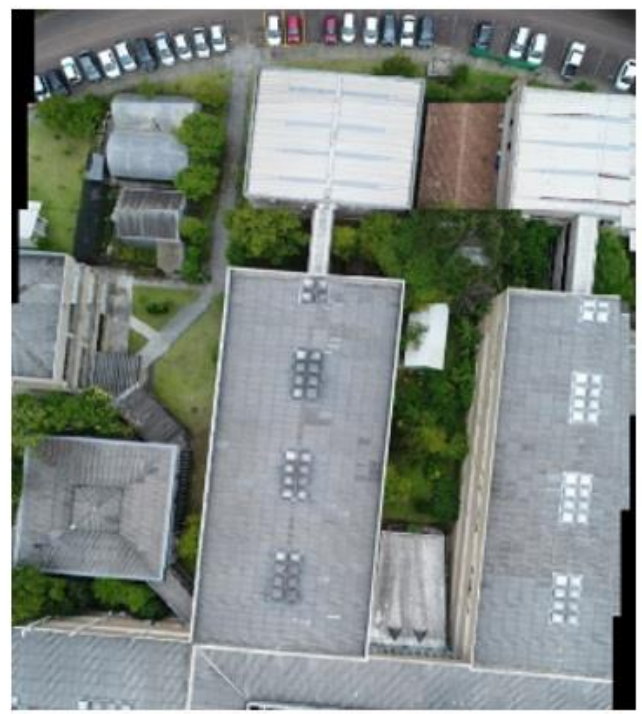

Fonte: Elaborada pelos autores.

A Figura 7 apresenta os mosaicos digitais produzidos com descontinuidades perceptíveis. Estas descontinuidades são frequentes e visíveis em mosaicos com mais de um par de imagens. A Figura 8 mostra as imagens de referência e de pesquisa usadas nos experimentos do Cenário III. 
Figura 8 - Pares de imagens do cenário III. Imagens de referência e de pesquisa para (a) Experimento 7. (b) Experimento 8
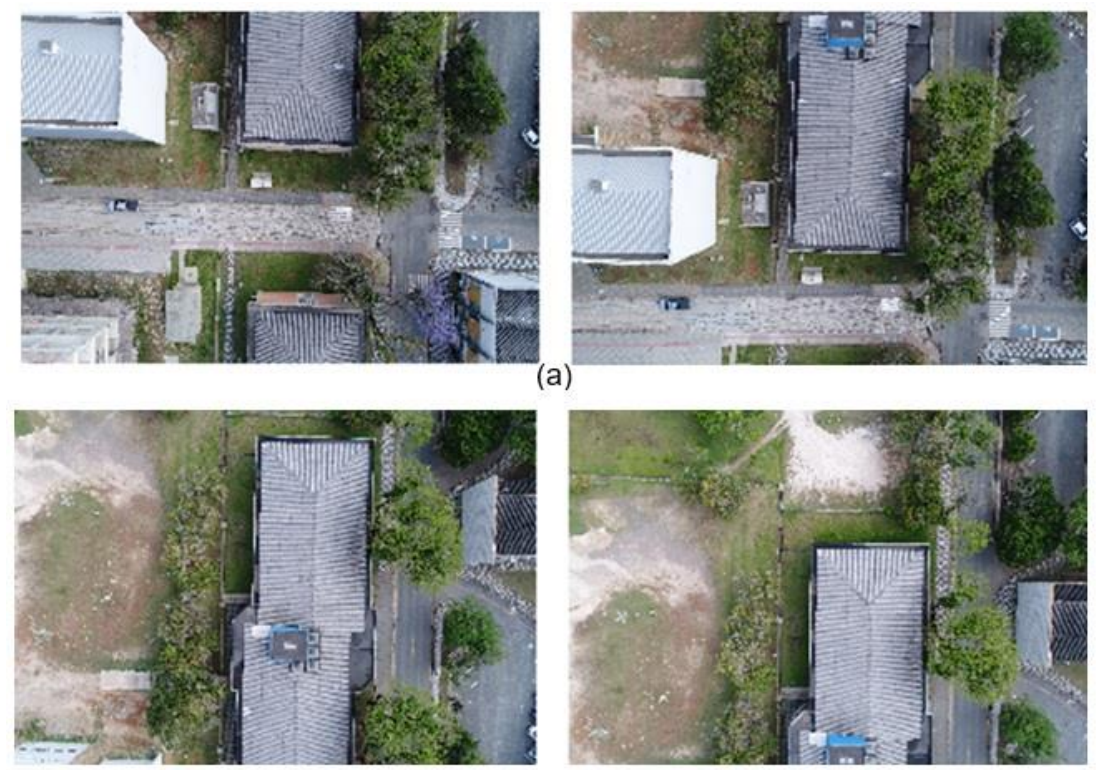

(b)

Fonte: Elaborada pelos autores.

A Figura 9 mostra os mosaicos digitais obtidos com o método proposto para cada experimento do Cenário III.

Figura 9 - Mosaicos digitais obtidos com o método proposto. (a) mosaico digital referente a par de imagens do experimento 7. (b) mosaico digital referente a par de imagens do experimento 8

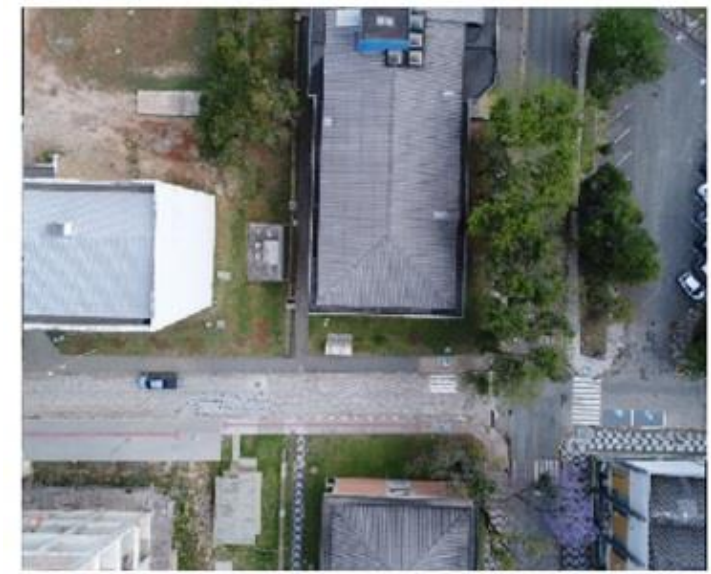

(a)

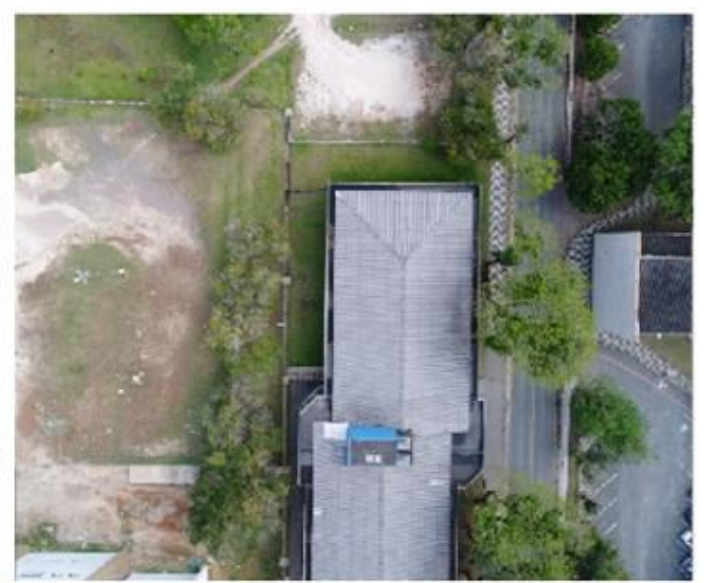

(b)

Fonte: Elaborada pelos autores. 
Na Figura 9 é mostrado os mosaicos digitais obtidos com o método proposto usando os pares de imagens dos experimentos 7 e 8 . Note que não há diferença radiométrica significativa e as inconsistências geométricas entre as linhas de junção das feições cartográficas não são perceptíveis. Para avaliar os benefícios do método proposto foi realizado dois experimentos usando como comparação os resultados obtidos com o método proposto e com o modelo ponto-a-ponto (método convencional) desenvolvido por Stegmann (2001). A Figura 10 mostra as imagens de referência e de pesquisa usadas neste experimento.

Figura 10 - (a) Imagens de referência e de pesquisa para o experimento de avaliação 1. (b) Imagens de referência e de pesquisa para o experimento de avaliação 2
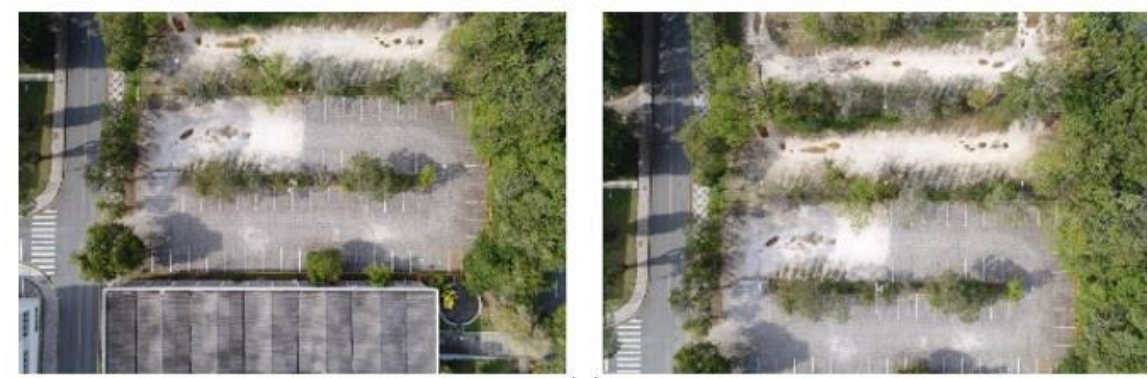

(a)
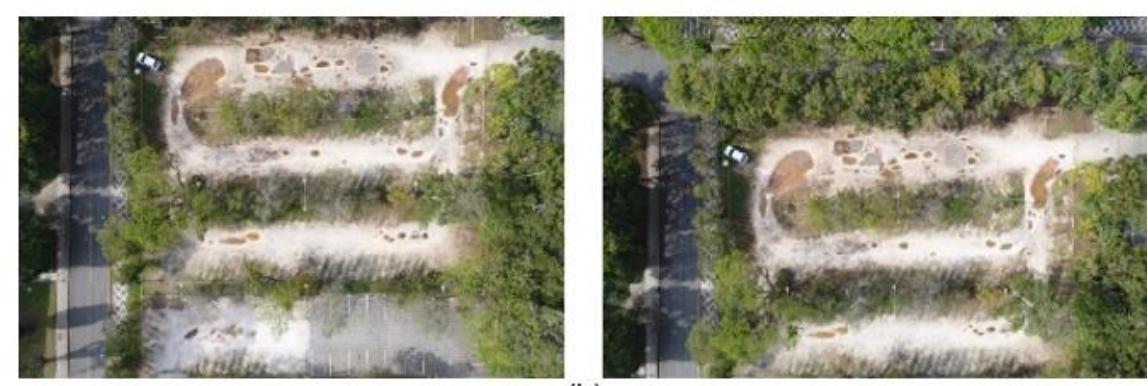

(b)

Fonte: Elaborada pelos autores.

A Figura 11 mostra os mosaicos digitais obtidos com o método de Stegmann (2001) e a Figura 12 mostra os resultados obtidos com o método proposto. 
Figura 11 - Resultado do mosaico digital obtido com o modelo de Stegmanan (2001). (a) Mosaico digital do experimento de avaliação 1. (b) Mosaico digital do experimento de avaliação 2. (c) Recorte do mosaico e ampliação das regiões do experimento de avaliação 2

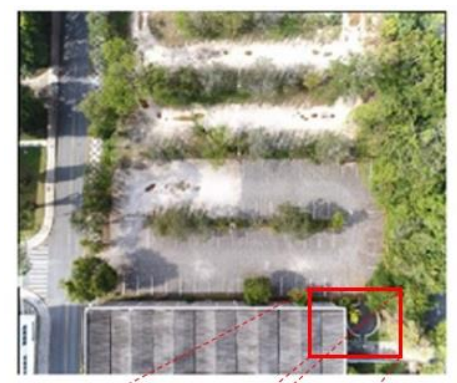

(a)

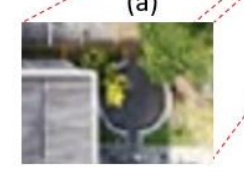

(c)

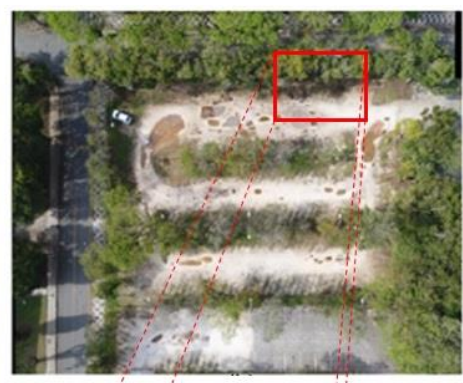

(b)

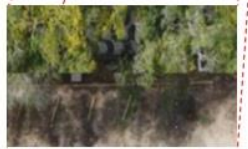

(d)

Fonte: Elaborada pelos autores.

Como pode ser visualmente observado nas Figuras 11(c)-11(d) e 12(c)12(d), o resultado obtido com o método de Stegmann (2001) e com o método proposto são compatíveis, isto é, ambos apresentam os mesmos valores de coeficientes de transformação e, em ambos os métodos, não são apresentados diferenças radiométricas significativas e descontinuidades não acentuada nas junções das feições. 
Figura 12 - Resultado dos mosaicos digitais obtidos com o modelo proposto. (a) Mosaico digital do experimento de avaliação 1. (b) Mosaico digital do experimento de avaliação 2. (c) Recorte do mosaico e ampliação das regiões do experimento de avaliação 1

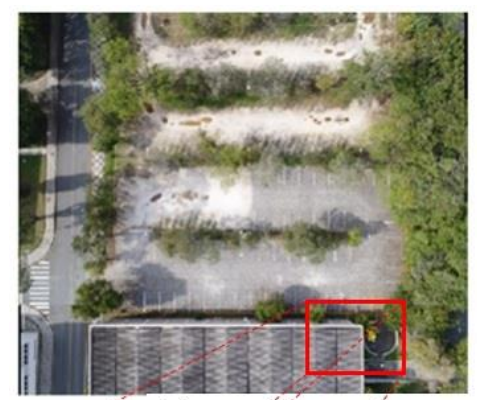

(a)

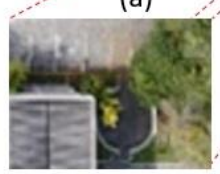

(c)

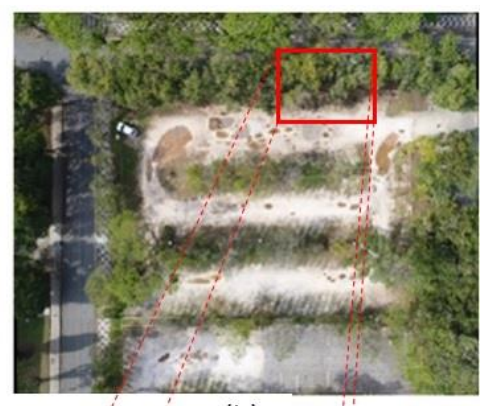

(b)

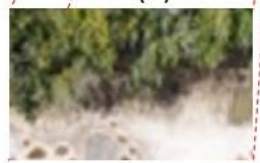

(d)

Fonte: Elaborada pelos autores.

\section{Conclusões e Recomendações}

Este trabalho apresentou um modelo para mosaicagem de imagens digitais baseado em uma abordagem ponto-a-reta. Experimentos com dados reais obtidos com uma plataforma RPA foram conduzidos. Os resultados obtidos mostraram que o modelo proposto é eficiente para geração de mosaicos digitais localmente consistentes. Quando comparado com o método convencional, a abordagem implementada neste trabalho apresentou resultados similares em termos de qualidade geométrica e radiométrica. Porém, pode-se observar que o método proposto é capaz de propiciar consistência geométrica, mas necessita de um algoritmo para balanceamento radiométrico das imagens. A matriz de construção do método proposto é capaz de detectar variações de escala entre as imagens. A técnica proposta para a estimativa dos parâmetros é relativamente simples. No entanto, pode ser aplicada somente em áreas urbanizadas. Como trabalhos futuros é recomendado maior investigação sobre o refinamento dos parâmetros de transformação usando um método de alinhamento global para produzir 
mosaicos de imagens digitais globalmente consistente e implementar um algoritmo de correspondência automática entre segmentos de retas.

\section{Agradecimentos}

O coautor do trabalho externa seus agradecimentos ao $\mathrm{CNPq}$ (processo: 303432/2016-0 PQ) pelo apoio concedido.

\section{Contribuição dos autores}

Este artigo foi desenvolvido a partir da pesquisa realizada no mestrado acadêmico do autor principal, sob orientação do coautor. O autor implementou o algoritmo e realizou os testes com dados reais. O coautor deste trabalho, desenvolveu o modelo matemático e contribuiu com a realização de testes e correções finais do texto.

\section{Referências}

ALI, S et al. Anisotropic motion estimation on edge preserving Riesz wavelets for robust video mosaicking. Pattern Recognit. Vol. 51, 2016, pp. 425-442.

ARRUDA JR, E. R. Mosaicagem de imagens digitais. Dissertação de mestrado. Universidade Estadual Paulista, Programa de Pós-Graduação em Ciências Cartográficas, 2002. 96p.

ARTERO, A. O. Técnicas para extração automática de feições retas em imagens digitais. Dissertação de mestrado. Universidade Estadual Paulista, Programa de Pós-Graduação em Ciências Cartográficas, 1999. 134p.

BERBERIDIS, K., KARYBALI, I. A new efficient cross-correlation based image registration technique with improved performance. In: Proceedings of Signal Processing Conference, 11th European. IEEE, 2002. pp. 1-4.

BUCHANAN, T. Critical sets for 3D reconstruction using lines. Computer Vision - ECCV. Berlin, Germany, 1992, pp.730-738. 
$\mathrm{CHO}, \mathrm{S}$. et al. Automatic Image Mosaic System Using Image Feature Detection and Taylor Series. In: Proceedings of DICTA, 2003, pp. 549-560.

DAL POZ, A. P. et al. Relational Matching Applied to Automatic Extraction of Ground Control in Digital Images. In: Proceedings of International Archives of Photogrammetry and Remote Sensing, Vienna, Austria, 1996, pp. 131134.

ELIBOL, A. et al. Fast topology estimation for image mosaicing using adaptive information thresholding. Robotics and Autonomous systems, vol. 61, n. 2, 2013, pp. 125-136.

GALO, M., TOZZI, C. L. Feature-point based matching: a sequential approach based on relaxation labeling and relative orientation. Journal of WSCG, vol.12, n.13, ISSN 1213-6972 WSCG'2004, February 2-6, 2004, Plzen, Czech Republic.

GAO, G., JIA, K. A new image mosaics algorithm based on feature points matching. In: Proceedings of Innovative Computing, Information and Control, 2007. ICICIC'07. Second International Conference on. IEEE, 2007, pp. 471471.

GHANNAM, S., ABBOTT, A. L. Cross correlation versus mutual information for image mosaicking. International Journal of Advanced Computer Science and Applications (IJACSA), December, vol. 4, n. 11, 2013.

GONZALEZ, R. C., WOODS, R.E. Processamento de imagens digitais. São Paulo: Editora Edgard Blucher, 2000. 524p.

HABIB, A. F., SCHENK, T. A new approach for matching surfaces from laser scanners and optical sensors, In: Proceedings of International Archives of Photogrammetry and Remote Sensing, vol. 32, 1999, pp. 55-61.

HABIB, A. Aerial triangulation using point and linear features. International Archives of Photogrammetry and remote Sensing, München, Germany, vol. 32, 1999, pp.137-141.

HABIB, A. F. et al. Photogrammetric and LiDAR data registration using linear features. Photogrammetric Engineering and Remote Sensing, vol. 71, n. 6, 2005, pp. 699-707.

HARTLEY, R. I., ZISSERMAN, A. Multiple View Geometry in Computer Vision. Cambridge University Press, ISBN: 0521623049, 2000. 
HASEGAWA, J. K., JUNIOR, E. R. A. Mosaico com imagens digitais. Boletim de Ciências Geodésicas, vol. 10, n. 1, 2004, pp. 31-50.

HEUVEL, F. A. van den. Exterior orientation using coplanar parallel lines. 10th Scandinavian Conference on Image Analysis, Lappeenranta, 1997, pp. 71-78.

ISLAM, B., KABIR, J. A new feature-based image registration algorithm. Computer Technology and Application, 2013, vol. 4, n. 2.

KLINEC, D. A model based approach for orientation in urban environments. In: Processamento de imagens digitais International Archives of Photogrammetry and Remote Sensing, commission III, Istanbul, 2004.

KUBIK, K., Relative and Absolute Orientation Based on Linear Features. Journal of Photogrammetry and Remote Sensing, vol. 46, 1998, pp. 199-204.

LI, M. et al. A study on automatic UAV image mosaic method for paroxysmal disaster. In: Proceedings of the International Society of Photogrammetry and Remote Sensing Congress, Melbourne, Australia. 2012, pp. 123-128.

LIU, Y. et al. Determination of camera locations from $2 \mathrm{D}$ to $3 \mathrm{D}$ line and point correspondences. IEEE Transactions on Pattern Analysis and Machine Intelligence, vol. 12, no. 1, 1990, pp.28-37.

LOWE, D. G. Distinctive image features from scale-invariant keypoints. International journal of computer vision, vol. 60, n. 2, 2004, pp 91-110.

MIKHAIL, E. M., ACKERMAN, F. Observations and least squares, New York, IAP. Dun-Donneley Pub, 1976.

MARQUES FILHO, O., NETO, H. V. Processamento digital de imagens. Brasport, 1999.

NEMRA, A., AOUF, N. Robust invariant automatic image mosaicing and super resolution for UAV mapping. In: Proceedings of Mechatronics and its Applications, 2009. ISMA'09. 6th International Symposium on. IEEE, 2009, pp. 1-7.

OLIVEIRA, H. C. et al. Surface Gradient Approach for Occlusion Detection Based on Triangulated Irregular Network for True Orthophoto Generation. IEEE Journal of Selected Topics in Applied Earth Observations and Remote Sensing. vol, 11. 2018, pp. 443-457. 
PATIDAR, D., JAIN, A. Automatic image mosaicking: an approach based on FFT.

International Journal of Scientific Engineering and Technology, vol. 1, n. 1, 2011, pp. 01-04.

PETSA, E., PATIAS, P. Sensor attitude determination using linear features. In: Proceedings of International Archives of Photogrammetry and Remote Sensing, vol. XXX, Commission I, 1994, pp.62-70.

ROBERTS, K. A New Representation for a Line. In: Proceedings of International Conference on Computer Vision and Pattern Recognition, 1988, Ann Arbor, MI, USA, pp. 635-640.

TOMMASELLI, A. M. G., LUGNANI, J. B. An alternative mathematical model to the collinearity equation using straight features. Proceedings of 16th International Congress of Photogrammetry and remote Sensing. Kyoto, 1988.

TOMMASELLI, A. M. G., TOZZI, C. A recursive approach to space resection using straight lines. Photogammetric Engineering and Remote Sensing, vol. 62, n. 1, 1996, pp. 55-66.

SAHOO, Prasanna K.; SOLTANI, S. A. K. C.; WONG, Andrew KC. A survey of thresholding techniques. Computer vision, graphics, and image processing, vol. 41, n. 2, 1988, pp 233-260.

SCHENK, T.. Digital photogrammetry: Vol. I: Background, fundamentals, automatic orientation producers. TerraScience, 1999.

SHAN, F. An approach to single image automatic orientation and point determination by using ortho-images and DTM. Photogrammetric Record. vol. 17, 2001, pp. 343-353.

STEGMANN, M. B., Image warping, Informatics and Mathematical Modeling, Technical University of Dinmark, 2001.

TIAN, G. Y. et al. Comprehensive interest point based imaging mosaic. Pattern Recognition Letters, vol. 24, n. 9-10, 2003, pp 1171-1179.

TIAN, Y. et al. Seam-line determination via minimal connected area searching and minimum spanning tree for UAV image mosaicking. International Journal of Remote Sensing, 2018, pp. 1-15. 
VENKATESWAR, V., CHELLAPPA, R. Extraction of straight lines in aerial images. IEEE Transactions on Pattern Analysis and Machine Intelligence, vol 14, n. 11, 1992, pp. 1111-1114.

XIA, M., et al. Globally consistent alignment for planar mosaicking via topology analysis. Pattern Recognition, vol. 66, 2017, pp 239-252.

XIAO, J. et al. Adaptive region-based video registration. In: Application of Computer Vision, 2005. WACV/MOTIONS'05 Volume 1. Seventh IEEE Workshops on. IEEE, 2005. pp. 215-220.

XING, C. et al. A robust method for mosaicking sequence images obtained from UAV. In: Information Engineering and Computer Science (ICIECS), 2010 2nd International Conference on. IEEE, 2010. pp. 1-4.

ZALMASON, G. Hierarchical recovery of exterior orientation from parametric and natural 3-D curves. In: International Archives of Photogrammetry and Remote Sensing, vol. XXXIII, part B2, p.610- 617, 2000.

ZHANG, C. Towards and operation system for automated updating for road database by integration of imagery and geodata. ISPRS Journal of Photogrammetry and Remote Sensing, vol. 58, 2004, pp. 166-186. 